\title{
Remote Control System Lab Based on the Technology of Ethernet
}

\author{
Juan Liu ${ }^{(1)}$ \\ Guangzhou vocational college of science and \\ technology, Guangzhou 510550, China, \\ E-mail: 20622414091@qq.com
}

$$
\text { Hongbo Qin }{ }^{(2)}
$$

Automation Company, Tangshan Iron and Steel Corporation, Tangshan 063000, China

E-mail: 37470540@qq.com

\begin{abstract}
In order to solve the contradiction between finite laboratory resource and ever-increasing students, a networking laboratory system based on serial communication server is designed and implemented. The campus network user can monitor the actual laboratory system through the serial relocation software or the IP address directly. And embedded TCP/IP is introduced simply.
\end{abstract}

Keywords-remote monitoring; TCP/IP; serial server; Ethernet

\section{INTRODUCTION}

With the rapid development of higher education, on the one hand, the number of students and the scale of universities enlarged ceaselessly. On the other hand, the education resource are not supplied effectively and timely. So the ever-increasing number of students and the relative deficiency of education resource, especially the relative deficiency of laboratory resource, has become a contradiction at the present stage. In addition, higher education experiments are lack of innovation, and students are lack of interest in prescriptive experiments of the school. How to make efficient and rational use of the existing experiment conditions to continue experiment teaching, improve students' enthusiasm, build innovative experiments, practice teaching condition and environment, scientific research will play a significance role to solve these problems.

This thesis proposes a concrete implementing scheme of open laboratory. According to actual experiment condition, using Ethernet and automation technology can realize remote access and monitor experimental fields through campus network even through the Internet. And it makes users feel like in site. So it will not only improve the laboratory resource utility, ease contradiction between finite laboratory resource and ever-increasing students effectively, but also improve technology content of experimental means of automation specialty, enlarge students' vision of specialty and will have a good effect on cultivating students' experiment interest too.

\author{
Zhengshi Chen ${ }^{(3)}$ \\ Guangzhou vocational college of science and \\ technology, Guangzhou 510550,China \\ E-mail: 2636444756@qq.com
}

\section{WHOLE SCHEME OF THE SYSTEM}

Most of electric and automation laboratory equipment have communication serial and can monitor and operate experimental process data by local computers. But they are lack of the function of connecting to the Ethernet or campus network. Taking process control laboratory as an example, based on communication interface of Ethernet monitoring system and software technology, this thesis carries out the second development and design to the laboratory equipment which have traditional communication serial, and realize remote and opening control system platform that is based on the construction of industrial Ethernet.

Taking the popular use of PCE-I process control system laboratory equipments in process control laboratory as an example, it mainly auto-adjustment controls physical analog of continuous industry process, such as liquid level, pressure, flow rate, temperature and other thermal parameters. Host computers use King View Software of Beijing Asia Control Automatic Software Company to realize the function of local data acquisition, flow control, animation display, report output, real-time and history data process, alarming and security mechanis $m$, trend curve and enterprise network monitoring.

Traditional laboratory equipment is connected with computer by RS-232 interface of console, and the maximu $\mathrm{m}$ communication distance is only 15 meters. So it cannot satisfy remote monitoring needs. Take use of the existing laboratory Ethernet system to connect serial laboratory equipment to network, then to campus network further. In terminal equipment, realize transformation between serial port and net mouth, and by using serial networking module, realize transformation from serial data to network data which support Ethernet TCP/IP protocol. The general block diagram of this system is shown in Fig. 1. 


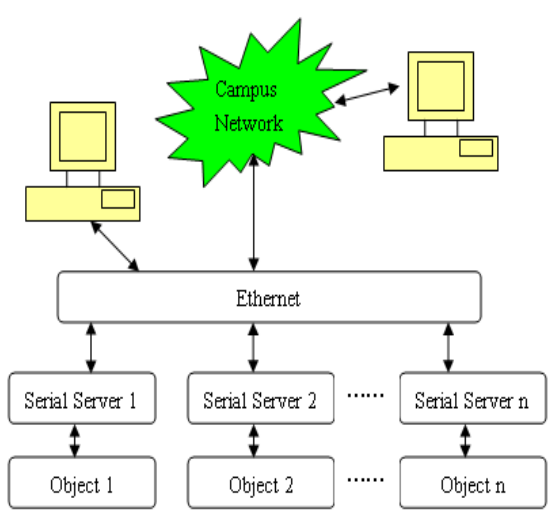

Figure 1. The structure diagram of Ethernet

About data acquisition part, devices of laboratory equipment themselves still can be used, such as liquid level sensor, pressure sensor, flow sensor, standard industrial automation instruments and so on. Signals complete A/D conversion through Newton 7000 serial module, and send the experiment data to RS-232 serial port. Host-computer users still use the original King View monitoring software. The use of relocation serial program will correspond local PC serial to remote laboratory equipment serial. As the King View software supports TCP/IP protocol, so by setting data resource on software, it can realize directly accessing the IP of the Ethernet and exchanging data with laboratory equipment.

\section{KEY TECHNOLOGY USED IN SYSTEM}

Computer technology, automation technology and network technology are used in the construction of Ethernet-based remote platform. There is no network mouth for laboratory system to connect with Ethernet, and only a standard RS-232 serial. So it is needed to develop and configurate intelligent network node on the bottom layer, exchange data between serials and network nodes, realize functions of sending serial data to network and receiving network command for laboratory equipment.

Because of all the already existing King View software support serial and TCP/IP network access, user end can access experimental end in two ways. One method is to install virtual serial driving program and create virtual serial. By parameter setting, build the corresponding relationship between virtual serial of computer and remote real serial. Users may use these virtual serials as reality ones. Another method is to set fixed IP address for the serial networking module of experimental terminal. Users access and control laboratory equipment by accessing IP address of the Ethernet.

\section{A. Serial server}

NP-311 serial networking server of Shenzhen Sanwang Communication Company is used on the bottom-layer intelligent network interface. It supports standard API interface, such as WINSOCK, and direct access es mode of TCP/UDP Ethernet. It has On-the-Fly no-setting function, and realizes real-time monitoring client software and realtime adjusting by driving program. It also supports TCP (Transmission Control Protocol), UDP (User Datagram Protocol), ARP (Address Resolution Protocol), ICMP (Internet Control Message Protocol), DHCP (Dynamic
Host Configuration Protocol) and Server\&Client model, making serial laboratory equipment working at C/S model.

This equipment module has On-the-Fly no-setting function, monitors users' software, real-time adjusts serial parameter, realizes a serial of parameter auto-setting, such as serial rate, parity check, bit and so on in the using process. It also provides secure password protection function, which is very useful in the open fieldbus structure and will prevent illegal user access and protect laboratory equipment.

\section{B. Embedded TCP/IP protocol}

Memories of embedded equipment are usually finite, so it will be very difficult to embed the whole TCP/IP protocol into the system. Actually, not all of the protocols will be used. So according to the feature of embedded systems and actual needs, simplified TCP/IP protocol family is chosen to instead of the whole. From $\mathrm{migh}$ to low, its architecture is divided into four parts: application layer, transport layer, network layer and link layer, each of which has a corresponding protocol set to complete different communication functions.

\begin{tabular}{|c|}
\hline Application Layer \\
(Telnet, FTP, HTTP, DNS, etc) \\
\hline Transport Layer (TCP, UDP) \\
\hline Network Layer (IP, ICMP, IGMP) \\
\hline Link Layer (Ethernet, Token Ring Network, \\
FDDI, etc) \\
\hline
\end{tabular}

Figure 2. The delamination of TCP/IP

As the lowest layer of TCP/IP protocol, link layer is also called data link layer or network interface layer. It is mainly used to receive IP packets of network layer and send them to serial laboratory equipment through physical interface. Or receive data frame from serial equipment, extract packet and send them to network layer. It also sends ARP request and receives ARP response for ARP module.

IP protocol is the main protocol of network layer and one of the most important layers of the whole protocol family. It is the basis of TCP, UDP, ICMP and IGMP. It provides unreliable and connectionless packet transfer service. In addition, ICMP on network layer is responsible for transferring error message and other information that need to be noticed. And it transfers on datagram inner; IGMP is used to support host and router to multicast, and make all systems be on physical network and know the multicast group of the current host.

Transport layer is mainly used for end-to-end communication between two host applications and it provides two different transport protocols: UDP (User Datagram Protocol) and TCP (Transport Control Protocol). UDP is a connectionless and unreliable transport protocol while TCP is a connection-oriented and reliable transport protocol. TCP host communicates with client through the 
three so-called "shake hand" certification and creates a virtual link path among networks except UDP. Therefore, there will be some UDP data packets losing phenomenon.

After serial server receives network data, it accesses to TCP/IP protocol and processes Ethernet data frame to judge if it is ARP packet, IP packet, ICMP packet, or TCP packet. Once be sure, get into correspond packet processing procedure. Interrupt mode is adopted to improve CPU utilization ratio in programming. Main program will enter interrupt program to process response when there is new data to receive or send. Network data processing flowchart is shown in Fig. 3.

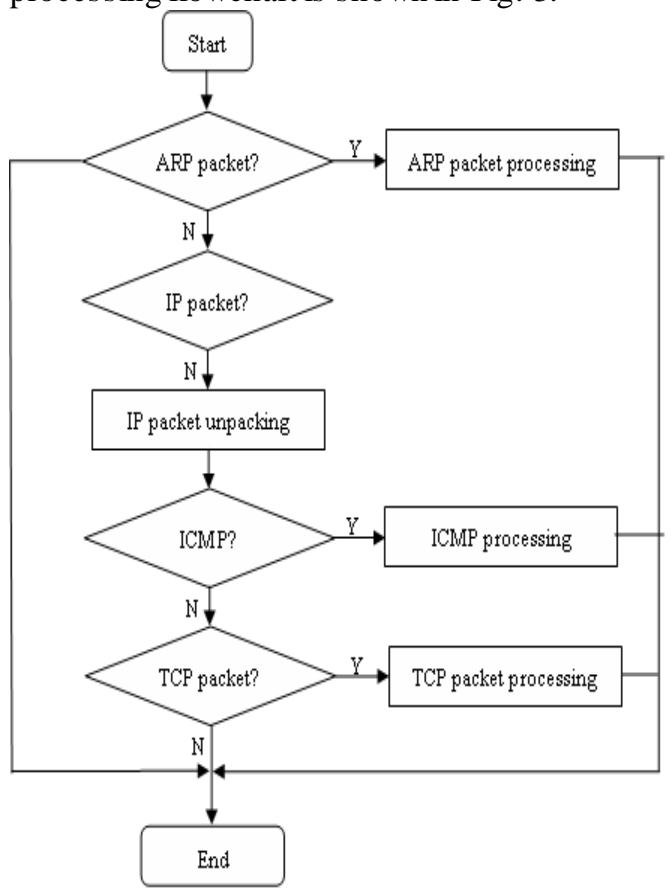

Figure 3. The flow chart of network data processing

\section{Host-computer software design}

In the user end, King View software which is based on Windows platform is used. It has the functions of fast constituting and generating host-computer monitor systems, and it is powerful open. So applications complied by development tools, such as VC, VB and so on, can access variables in real-time database of King View and control King View.

King View software supports lots of network architecture functions, such as TCP/IP, 485/232 and so on. So it provides more choice to realize remote monitoring. One method is accessing the uniquely determined IP address of serial server to realize network control. Furthermore, a kind of virtual serial software, which is also called serial relocation software, is installed to the user end to correspond reality serials of laboratory equipment to virtual serials of user end. It forms a network mapping to the reality serial. Then it will realize laboratory equipment networking access and control through King View software of userend.

Moreover, laboratory systems which have no general software of host-computer, can realize dynamic data exchange between remote experimental user server and local lab through remote ActiveX control that based on
Socket remote experiment or remote experimental Java Applet program. Both ActiveX control and Java Applet resident in Web server, and users download to remote client before doing experiments. Diffe rences between them is that after ActiveX control downloading to remote Client, Socket connection is built directly to remote experimental proxy server that has independent IP address, realizing dynamic experiment data transport and it doesn't need to pass Web server. Yet, when using Java Applet technology to realize dynamic data exchange, the small program that is downloaded to remote experimental station creates Socket connection with Web server. Web server connects to remote proxy server in LAN, and both dynamic experiment data and control command exchange between remote experimental stations and remote experimental proxy servers need to relay by Web server.

\section{CONCLUSIONS}

Building laboratory Ethernet system makes a breakthrough to the request of lab on time and space. Especially for these equipment that are fairly expensive or need repeatedly test, it is of great significance. Laboratory equipment in one campus can be operated by different campuses. And it will play an important role in the cross regional cooperation of different universities and research units.

In addition, choosing remote experimental Ethernet that uses serial networking server will achieve remote laboratory effect without changing original laboratory condition too much.

\section{REFERENCE}

[1] Wang Miaomiao ,The Design of Oil Field Remote Monitoring System based on Improved B/S Structure.Proceedings of 2011 International Conference on Engineering and Information Management (ICEIM2011).

[2] Yifeng Hou. Research on Remote Monitoring and Control System Based on Communication Technology by Power Line Carrier[A]. Selected,Peer Reviewed Papers from the 2013 2nd International Conference on Mechanical Engineering,Industrial Electronics and Informatization(MEIEI 2013)

[3] Zewen Wang, Wei Li, Design of the Remote Monitoring System for Mine Hoists,The 24th Chinese control conference proceedings and decisions 2013

[4] Qian Li-biao, Yang Ma-ying, Yu Li, etc. Remote Control Laboratory System Base on Web and S7-300PLC. Journal of Zhejiang Industrial University. 2007.2,

[5] G. P. Liu,J. X. Mu,D. Rees and S. C. Chai.Design and stability analy sis of net worked control systems with random communication time delay using the modified MPC. International Journal of Control. 2006

[6] Xian-qiu wu, chao-hui liu, Under the net work environment of the remote experiment technology study [J]. Journal of experimental technology and management. 2008 (6)

[7] Ma Aimei. Remote experiment of technical analysis and system construction $[\mathrm{J}]$. Journal of information science and technology. $2012(10)$

[8] WANG X Y,DAI Y P,XU X Y.Design of networked control experiment equipment based on three-container water tank. Proceedings of the Fourth China-Japan International Workshop on Internet Technology and Con-trol Applications . 2005

[9] teaching fang, GuHuiJuan Wang Jian. An open Internet remote experiment device and development [J]. 2011 (04) laboratory research andexploration.

[10] ying-xu lai, li jian, Based on the platform of network teaching experimental design Free - 1 [J]. 2011 (11) laboratory research and exploration. 